\title{
EXTREMAL ANALYTIC DISCS WITH PRESCRIBED BOUNDARY DATA
}

\author{
CHIN-HUEI CHANG, M. C. HU AND HSUAN-PEI LEE
}

\begin{abstract}
This paper concerns the existence and uniqueness of extremal analytic discs with prescribed boundary data in a bounded strictly linearly convex domain $D$ in $\mathbf{C}^{n}$. We prove that for any two distinct points $p, q$ in $\partial D$ (respectively, $p \in \partial D$ and a vector $v$ such that $\sqrt{-1} v \in T_{p}(\partial D)$ and $\langle v, \bar{\nu}(p)\rangle=\sum_{1}^{n} v_{j} \bar{\nu}_{j}(p)>0$ where $\nu(p)$ is the outward normal to $\partial D$ at $\left.p\right)$ there exists an extremal analytic disc $f$ passing through $p, q$ if $\partial D \in C^{k}, k \geq 3$ (respectively, $f(1)=p, f^{\prime}(1)=v$ if $\partial D \in C^{k}, k \geq 14$ ). Consequently, we can foliate $\bar{D}$ with these extremal analytic discs.
\end{abstract}

Introduction. In this paper, we always assume that $D$ is a bounded strictly linearly convex domain in $\mathbf{C}^{n}$ (see $\S 1$ for definitions). Let $U$ denote the open unit disc in C. An extremal analytic disc $f$ of $D$ is a holomorphic map $f$ from $U$ into $D$ such that the Kobayashi distance of any two points in $f(U)$ is realized by $f$. Lempert in $[6,7]$ has proved the existence, uniqueness and boundary regularity of such maps with data given at interior points. Following [7] we shall use the term "E-mapping" for the "extremal analytic disc." It is also known [6, Proposition 5] that if $\partial D$ is of class $C^{k}, k \geq 3$, then any $E$-mapping of $D$ is in $C^{k-2}(\bar{U})$.

Our first result is as follows:

THEOERM 1. Suppose that $\partial D$ is of class $C^{k}, k \geq 3$. Then, for any two distinct points $p, q \in \bar{D}$, there exists an E-mapping $f: \bar{U} \rightarrow \bar{D}$ with $p, q \in f(\bar{U})$. Any other $E$-mapping passing through $p, q$ differs from $f$ by a unique automorphism of $U$.

The existence is proved by establishing a uniform $\frac{1}{4}$-Hölder estimate for $E$ mappings of $D$ when they are suitably parametrized. Here, "parametrize" means composing the $E$-mapping with an automorphism of $U$. The uniqueness has already been proved in [7]. In $\S 1$ we exhibit an extremal property of the $E$-mapping $f$ when at least one of $p, q$ is on $\partial D$ (see Proposition 1). The extremal property of $f$ when both $p, q$ are interior points is already known in [6].

Theorem 2 is the infinitesimal version of Theorem 1 when $p, q$ tend to a boundary point.

THEOREM 2. Suppose that $\partial D$ is of class $C^{k}, k \geq 14$. Let $p$ be a fixed point on $\partial D, \sqrt{-1} v \in T_{p}(\partial D)$, with $\langle v, \bar{\nu}(p)\rangle>0$, where $\nu(p)$ denotes the outward normal to $\partial D$ at $p$ and $\langle u, w\rangle=\sum_{i=1}^{n} u_{j} w_{j}$ for $u, w \in \mathbf{C}^{n}$. Then there exists an E-mapping

Received by the editors September 1, 1987 .

1980 Mathematics Subject Classification (1985 Revision). Primary 32H15.

The first and third authors were partially supported by NSC Grant No. NSC76-0208-M001-07. The second author was partially supported by NSC Grant No. NSC76-0208-M008-08. 
$f: \bar{U} \rightarrow \bar{D}$ such that $f(1)=p, f^{\prime}(1)=v$. Any other E-mapping $g$ of $D$ with $g(1)=p, g^{\prime}(1)=v$ differs from $f$ by an automorphism of $U$.

If we require the $E$-mapping $f$ in Theorem 2 to have a special parametrization, then $f$ is uniquely determined by an extremal property (see Proposition 3 ). The idea for proving the existence part of Theorem 2 is the same as that of Theorem 1: We employ known results from [6, 7] to obtain a sequence of $E$-mappings, and then we show that we can extract a subsequence converging to the desired "nondegenerate" $E$-mapping. So the uniform $\frac{1}{4}$-Hölder estimate used in proving Theorem 1 is also needed in proving Theorem 2, but the situation in this case is more complicated. When the boundary of $D$ at $p$ is real analytic, by using Vitushkin's partial normal form, we can easily show that the limit is nondegenerate; for the general case, we construct a family of domains which are small perturbations of a real analytic domain. We then prove the existence of $E$-mappings under small perturbations to conclude the proof of Theorem 2. It is here that we need $\partial D \in C^{k}, k \geq 14$. The Vitushkin's normal form for a real analytic strongly pseudoconvex hypersurface is a variant of Chern-Moser's normal form (cf. Vitushkin [10] or Chang-Lee-Wang [1]).

As a consequence of Theorems 1 and 2, we have the following:

THEOREM 3. Suppose that $\partial D \in C^{k}, k \geq 14$, and there is a continuous map $\Phi: \partial D \times \bar{D} \rightarrow \bar{B}_{n}$ where $B_{n}=\left\{z \in \mathbf{C}^{n}|| z \mid<1\right\}$. For $p \in \partial D$ fixed, the map $\Phi(p, \cdot): \bar{D} \rightarrow \bar{B}_{n}$ is a homeomorphism and $C^{k-5}$ in $\bar{D} \backslash\{p\}$. In fact,

$$
\Phi \in C^{k-5}(\partial D \times \bar{D} \backslash\{\text { diagonal of } \partial D \times \partial D\}) .
$$

When $\partial D \in C^{k}, k \geq 3$, the boundary curves of the $E$-mappings are biholomorphic invariants of $D$. When $\partial D$ is real analytic, their boundary curves, in general, are different from the Chern-Moser chains (see [1]). Indeed, the chains are determined locally while the $E$-mappings are not. When $D$ is not strongly pseudoconvex, the boundary regularity of an $E$-mapping is still not clear (cf. Poletskii [8] and Suzuki [9]).

PLAN OF THE PAPER. $§ 1$ includes some definitions and previous results from $[6$, 7], as well as the proof of the uniqueness part of Theorem 2 and extremal properties of $E$-mappings. $\S 2$ consists of the Hölder estimate for $E$-mappings and the proof of the existence part of Theorem 1 . In $\S 3$ we prove the existence part of Theorem 2 while the proof of the existence of $E$-mappings under small perturbation of domains is detained till $\S 4$. Theorem 3 is proved in $\S 5$.

ACKnowledgment: We are grateful to Professors T. C. Lee and A. N. Wang for many fruitful discussions.

\section{Definitions, extremal properties and the uniqueness part of Theo-} rem 2. A domain $D$ in $\mathbf{C}^{n}$ is said to be linearly convex if through any boundary point $p$ of $D$ there passes a complex hyperplane that is disjoint from $D$. A domain $D$ in $\mathbf{C}^{n}$ is called strictly linearly convex if it has a $C^{2}$ boundary and it remains linearly convex under small $C^{2}$ perturbation. If $\rho$ is a defining function of a strictly linearly corrvex domain $D$ and $p \in \partial D$ then

$$
\sum_{j, k=1}^{n} \rho_{z_{j} z_{k}}(p) v_{j} \bar{v}_{k}>\left|\sum_{j, k=1}^{n} \rho_{z_{j} z_{k}}(p) v_{j} v_{k}\right|
$$

for every $v$ in the complex tangent space of $T_{p}(\partial D)$. 
For the rest of this paper $D$ will denote a bounded strictly linearly convex domain in $\mathbf{C}^{n}$. Suppose that $\partial D \in C^{k}, k \geq 3$. According to [6, 7], for an $E$-mapping $f$ of $D$ there exists a $C^{k-2}$ map $\tilde{f}$ from $\bar{U}$ into $\mathbf{C}^{n}$, holomorphic in $U$ and

$$
\left.\tilde{f}(\varsigma)\right|_{\partial U}=\varsigma p(\varsigma) \bar{\nu}(f(\varsigma)),
$$

where $p(\varsigma)$ is a $C^{k-2}$ function from $\partial D$ into $\mathbf{R}^{+}$and $\nu(f(\varsigma))$ denotes the outward normal to $\partial D$ at $f(\varsigma)$. In particular, we can choose $p(\varsigma)=\left\langle\varsigma f^{\prime}(\varsigma), \bar{\nu}(f(\varsigma))\right\rangle^{-1}$ and we have $\left\langle f^{\prime}(\varsigma), \tilde{f}(\varsigma)\right\rangle \equiv 1$ which we will always assume.

A subset $R$ of $D$ is called a holomorphic retract of $D$ if there exists a holomorphic map $r$ from $D$ into $R$ such that $r(z)=z$ for $z \in R$. It is known (see [7]) that complex one-dimensional retracts of $D$ are exactly the images of $E$-mappings of $D$. Thus if $f$ is an $E$-mapping of $D$ we can find a map $F: \bar{D} \rightarrow \bar{U}$ holomorphic in $D$ such that $F \circ f=\operatorname{id}_{\bar{U}}$. The map $F$ will be called the left inverse of $f$.

Lemma 1. Assume that $f: \bar{U} \rightarrow \bar{D}$ is holomorphic in $U, C^{1}$ in $\bar{U}$. If, for some $\theta_{0} \in \mathbf{R}, f\left(e^{i \theta}\right) \in \partial D$ for $\theta \in \mathbf{R}$ near $\theta_{0}$ then

$$
\left\langle f^{\prime}\left(e^{i \theta_{0}}\right), e^{i \theta_{0}} \bar{\nu}\left(f\left(e^{i \theta_{0}}\right)\right)\right\rangle \geq 0 .
$$

Proof. Inequality (1) is obvious if $\partial D$ is strictly convex near $f\left(e^{i \theta_{0}}\right)$. The lemma follows by noting that the product on the left-hand side of (1) is invariant under local biholomorphism in a neighborhood of $f\left(e^{i \theta_{0}}\right)$.

REMARK 1 . Lemma 1 shows that the assumption in Theorem 2 is necessary.

The following Lemmas 2, 3 appeared in Lempert [7]. Here we give different proofs and use them to prove Proposition 1 concerning the extremal properties.

Lemma 2. Let $\varphi: \bar{U} \rightarrow \bar{U}$ be holomorphic in $U, C^{1}$ on $\bar{U}$. Suppose that $\varphi(0)=$ $0, \varphi(1)=1$. Then $\varphi^{\prime}(1) \geq 1$ and the equality holds if and only if $\varphi=\operatorname{id}_{U}$.

Proof. Suppose that $\varphi \neq \mathrm{id}_{U}$. By the convexity of $U$ we have

$$
\operatorname{Re}(\varsigma-\varphi(\varsigma)) \bar{\zeta}>0 \text { for almost every } \varsigma \in \partial U .
$$

Since $\zeta /(\varsigma-1)^{2}<0$ for $\zeta \in \partial U \backslash\{1\}$ we have

$$
\operatorname{Re} \frac{\varsigma-\varphi(\varsigma)}{(\varsigma-1)^{2}}<0 \text { for almost every } \varsigma \in \partial U
$$

Hence

$$
\text { Rep.v. } \frac{1}{\pi i} \int_{|\varsigma|=1} \frac{\varsigma-\varphi(\varsigma)}{(\varsigma-1)^{2}} \frac{d \zeta}{\zeta}<0 .
$$

Plemelj's formula and Lemma 1 then give

$$
\varphi^{\prime}(1)=\operatorname{Re} \varphi^{\prime}(1)>1 .
$$

LEMMA 3. Let $\varphi: \bar{U} \rightarrow \bar{U}$ be holomorphic in $U$ and $C^{1}$ on $\bar{U}$. Suppose that $\varphi(1)=1, \varphi(-1)=-1$. Then $\left(\varphi^{\prime}(1)+\varphi^{\prime}(-1)\right) \geq 2$ and the equality holds if and only if $\varphi=\mathrm{id}_{U}$.

PROOF. We proceed exactly as in the proof of Lemma 2 except that we multiply $\operatorname{Re}(\zeta-\varphi(\varsigma)) \bar{\zeta}>0$ by $\varsigma^{2} /(\zeta-1)^{2}(\zeta+1)^{2}$ which is negative on $\partial D \backslash\{\zeta= \pm 1\}$.

Using Lemmas 2, 3 we can prove the uniqueness part of Theorem 1. Since it has already been done in [7] we will not repeat it here. The next lemma concerns the relation between $F$ and $\tilde{f}$ of an $E$-mapping $f$ of $D$. 
Lemma 4. Suppose that $\partial D$ is of class $C^{k}, k \geq 3$. Let $f$ be an E-mapping of $D$ with corresponding $\tilde{f}$ and left inverse $F$. Then $\tilde{f}(\varsigma)=\left(F_{z_{1}}(f(\varsigma)), \ldots, F_{z_{n}}(f(\varsigma))\right)$ for all $\varsigma \in U$ where $F_{z_{j}}=\partial F / \partial z_{j}$. Hence $F$ is in $C^{k-1}(\bar{D})$.

ProOF. For $z \in D$, by Lempert [7, p. 345] we have

$$
\langle z-f(F(z)), \tilde{f}(F(z))\rangle=0 .
$$

Differentiating the above formula with respect to $z_{j}$, we obtain

$$
\sum_{k=1}^{n}\left(\delta_{j k}-f_{k}^{\prime}(F(z)) F_{z_{j}}(z)\right) \tilde{f}_{k}(F(z))+\left\langle z-f(F(z)), F_{z_{j}}(z) \tilde{f}^{\prime}(F(z))\right\rangle=0,
$$

which can be written as

$$
\begin{aligned}
\tilde{f}_{j}(F(z)) & =\left(\left\langle f^{\prime}(F(z)), \tilde{f}(F(z))\right\rangle-\left\langle z-f(F(z)), \tilde{f}^{\prime}(F(z))\right\rangle\right) F_{z_{j}}(z) \\
& =\left(1-\left\langle z-f(F(z)), \tilde{f}^{\prime}(F(z))\right\rangle\right) F_{z_{j}}(z) .
\end{aligned}
$$

For $z=f(\varsigma), \varsigma \in U$, we have $z-f(F(z))=f(\varsigma)-f(\varsigma)=0$. Hence

$$
\tilde{f}(\varsigma)=\left(F_{z_{1}}(f(\varsigma)), \ldots, F_{z_{n}}(f(\varsigma))\right. \text {. }
$$

From Lemma 4 and by applying Lemmas 2 and 3 with $\varphi=F \circ g$ we obtain

PROPOSITION 1. Suppose that the boundary of $D$ is of class $C^{k}, k \geq 3$. Let $f$ be an E-mapping of $D$ with corresponding $\tilde{f}$. Let $g$ be any analytic disc from $U$ into $D, C^{1}$ on $\bar{U}$ such that $f(0)=g(0), f(1)=g(1)(f(1)=g(1), f(-1)=g(-1)$ resp.). Then

$$
\begin{aligned}
& \left\langle g^{\prime}(1), \tilde{f}(1)\right\rangle \geq 1, \\
& \left\langle g^{\prime}(1), \tilde{f}(1)\right\rangle+\left\langle g^{\prime}(-1), \tilde{f}(-1)\right\rangle \geq 2, \quad \text { resp. }
\end{aligned}
$$

The equality holds if and only if $f \equiv g$.

REMARK 2. Without loss of generality we may assume in $(2)^{\prime}$ that

$$
\left\langle g^{\prime}(-1), \tilde{f}(-1)\right\rangle=1 .
$$

This can be achieved by composing $g$ with an automorphism of $U$. Thus $(2)^{\prime}$ will be of the same form as (2).

The rest of this section is devoted to the proof of the uniqueness part of Theorem 2 .

LEMMA 5. Let $\varphi: \bar{U} \rightarrow \bar{U}$ be holomorphic in $U, C^{3}$ on $\bar{U}$ such that $\varphi(1)=1$, $\varphi^{\prime}(1)=1, \varphi^{\prime \prime}(1)=0$. Then $\operatorname{Re} \varphi^{\prime \prime \prime}(1) \leq 0$ and the equality holds if and only if $\varphi=\operatorname{id}_{U}$.

ProOF. Suppose that $\varphi \neq \mathrm{id}_{U}$. We proceed as in the proof of Lemma 2, but we multiply $\operatorname{Re}(\varsigma-\varphi(\varsigma)) \bar{\zeta}>0$ by $\varsigma^{2} /(\varsigma-1)^{4}$ instead. Therefore for almost every $\varsigma \in \partial U$ we have

$$
\operatorname{Re} \frac{(\varsigma-\varphi(\varsigma)) \varsigma}{(\varsigma-1)^{4}}>0
$$

It follows that

$$
\text { Re p.v. } \frac{1}{\pi i} \int_{|\zeta|=1} \frac{\zeta-\varphi(\zeta)}{(\zeta-1)^{4}} d \zeta>0
$$

and Plemelj's formula gives $\operatorname{Re} \varphi^{\prime \prime \prime}(1)<0$. 
LEMMA 6. Suppose that $\partial D \in C^{k}, k \geq 2$. Let $f, g: \bar{U} \rightarrow \bar{D}$ be holomorphic in $U, C^{2}$ in $\bar{U}$. Suppose that there is a neighborhood $V$ of 1 in $\mathbf{C}$ such that $f(\partial U \cap V) \subset$ $\partial D$ and $g(\partial U \cap V) \subset \partial D$. If $f(1)=g(1)=p, f^{\prime}(1)=g^{\prime}(1)=v,\langle v, \bar{\nu}(p)\rangle>0$, then there is an $M \in \operatorname{Aut}(U)$ such that for $\tilde{G}=g \circ M$ we have $\tilde{G}(1)=p, \tilde{G}^{\prime}(1)=v$ and $\left\langle f^{\prime \prime}(1), \bar{\nu}(p)\right\rangle=\left\langle\tilde{G}^{\prime \prime}(1), \bar{\nu}(p)\right\rangle$.

PROOF. Since the equality $\left\langle f^{\prime \prime}(1), \bar{\nu}(p)\right\rangle=\left\langle\tilde{G}^{\prime \prime}(1), \bar{\nu}(p)\right\rangle$ is invariant under local biholomorphisms in a neighborhood of $p$, we may assume that $\partial D$ at $p$ is strongly convex at $p$ (i.e., the real Hessian of $\partial D$ at $p$ is positive definite). Claim:

$$
\operatorname{Re}\left\langle f^{\prime \prime}(1), \bar{\nu}(p)\right\rangle=\operatorname{Re}\left\langle g^{\prime \prime}(1), \bar{\nu}(p)\right\rangle .
$$

In fact, by the convexity of $\partial D$ at $p$, we have

$$
\operatorname{Re}\langle f(\varsigma)-g(\varsigma), \bar{\nu}(f(\varsigma))\rangle \geq 0 \text { for } \varsigma \in \partial U \cap V^{\prime},
$$

where $V^{\prime} \subset V$ is a neighborhood of 1 in $\mathbf{C}$ such that $f\left(\partial U \cap V^{\prime}\right)$ and $g\left(\partial U \cap V^{\prime}\right)$ are contained in a neighborhood of $p$ in $\partial D$ where it is strongly convex. So

$$
\operatorname{Re}\left\langle(g(\varsigma)-f(\varsigma)) /(\varsigma-1)^{2}, \varsigma \bar{\nu}(f(\varsigma))\right\rangle \geq 0 \text { for } \varsigma \in \partial U \cap V^{\prime}
$$

As $\zeta \rightarrow 1$ we obtain

$$
\operatorname{Re}\left\langle g^{\prime \prime}(1)-f^{\prime \prime}(1), \bar{\nu}(p)\right\rangle \geq 0 .
$$

Exchanging $f$ and $g$ we also obtain

$$
\operatorname{Re}\left\langle f^{\prime \prime}(1)-g^{\prime \prime}(1), \bar{\nu}(p)\right\rangle \geq 0 .
$$

From these two inequalities we obtain (3).

Now let $M(\varsigma)=e^{i \alpha}(\varsigma-a) /(1-\bar{a} \varsigma), \alpha \in \mathbf{R}, a \in U$, satisfying $M(1)=M^{\prime}(1)=1$. For such an $M \in \operatorname{Aut}(U)$ we have $(g \circ M)(1)=g(1),(g \circ M)^{\prime}(1)=g^{\prime}(1)$ and $(g \circ M)^{\prime \prime}(1)=g^{\prime \prime}(1)+2 \bar{a} g^{\prime}(1) /(1-\bar{a})$. It is easy to see that for such $a, 2 \bar{a} /(1-\bar{a})$ ranges over all purely imaginary numbers. Since $g^{\prime}(1)=v$ and $\langle v, \bar{\nu}(p)\rangle>0$, together with (3), we can find an $a \in U$ such that

$$
\left\langle f^{\prime \prime}(1), \bar{\nu}(p)\right\rangle=\left\langle(g \circ M)^{\prime \prime}(1), \bar{\nu}(p)\right\rangle .
$$

From the computations in the proof of Lemma 6 we have

Proposition 2. Let $\partial D \in C^{k}, k \geq 2$. Suppose that $f, g: \bar{U} \rightarrow \bar{D}$ are holomorphic in $U, C^{2}$ on $\bar{U}, f(1)=g(1)=p \in \partial D$ and $f^{\prime}(1)=g^{\prime}(1)=v$. Then we can find $M_{1}, M_{2} \in \operatorname{Aut}(U)$ such that for $\tilde{F}=f \circ M_{1}, \tilde{G}=g \circ M_{2}$ we have $\tilde{F}(1)=\tilde{G}(1)=p, \tilde{F}^{\prime}(1)=\tilde{G}^{\prime}(1)=v$ and $\operatorname{Im}\left\langle\tilde{F}^{\prime \prime}(1), \bar{\nu}(p)\right\rangle=\operatorname{Im}\left\langle\tilde{G}^{\prime \prime}(1), \bar{\nu}(p)\right\rangle=0$. Also if there is a neighborhood $V$ of 1 in $\mathbf{C}$ such that $f(\partial U \cap V) \subset \partial D$, then we have $\left\langle\tilde{F}^{\prime \prime}(1), \bar{\nu}(p)\right\rangle \leq\left\langle\tilde{G}^{\prime \prime}(1), \bar{\nu}(p)\right\rangle$.

Now we can prove the

Uniqueness part of Theorem 2. Let $f, g$ be two $E$-mappings of $D$ with $f(1)=$ $g(1)=p, f^{\prime}(1)=g^{\prime}(1)=v$. By Lemma 6 and Proposition 2 we may assume that $\left\langle f^{\prime \prime}(1), \bar{\nu}(p)\right\rangle=\left\langle g^{\prime \prime}(1), \bar{\nu}(p)\right\rangle \in \mathbf{R}$. Let $F, G$ be the left inverses of $f, g$ respectively. Then as $F \circ f=G \circ g=\mathrm{id}_{U}$ both the maps $F \circ g, G \circ f: \bar{U} \rightarrow \bar{U}$ satisfy the assumptions of Lemma 5 . On the other hand, as $\tilde{f}(\zeta)=\bar{\nu}(f(\varsigma))\left\langle f^{\prime}(\varsigma), \bar{\nu}(f(\varsigma))\right\rangle^{-1}$ 
on $\partial U$ and similarly for $\tilde{g}$ we see that $\tilde{f}(1)=\tilde{g}(1)$ and $\tilde{f}^{\prime}(1)=\tilde{g}^{\prime}(1)$ by our assumptions on $f$ and $g$. Note that from Lemma 4 we have

$$
\sum_{k=1}^{n} F_{z_{j} z_{k}}(f(\varsigma)) f_{k}^{\prime}(\varsigma)=\tilde{f}_{j}^{\prime}(\varsigma) \text { and } \sum_{k=1}^{n} G_{z_{j} z_{k}}(g(\varsigma)) g_{k}^{\prime}(\varsigma)=\tilde{g}_{j}^{\prime}(\varsigma) \text {, }
$$

$j=1, \ldots, n, \varsigma \in \bar{U}$. If $f \neq g$, Lemma 5 and an easy computation give

$$
\operatorname{Re}(F \circ g)^{\prime \prime \prime}(1)=\operatorname{Re}\left\{\left\langle g^{\prime \prime \prime}(1)-f^{\prime \prime \prime}(1), \tilde{f}(1)\right\rangle+3\left\langle g^{\prime \prime}(1)-f^{\prime \prime}(1), \tilde{f}^{\prime}(1)\right\rangle\right\}<0 .
$$

But we also have

$$
\operatorname{Re}(G \circ f)^{\prime \prime \prime}(1)=\operatorname{Re}\left\{\left\langle f^{\prime \prime \prime}(1)-g^{\prime \prime \prime}(1), \tilde{g}(1)\right\rangle+3\left\langle f^{\prime \prime}(1)-g^{\prime \prime}(1), \tilde{g}^{\prime}(1)\right\rangle\right\}<0 .
$$

As we have shown that $\tilde{f}(1)=\tilde{g}(1)$ and $\tilde{f}^{\prime}(1)=\tilde{g}^{\prime}(1)$, the above two inequalities can not hold simultaneously. This completes the proof.

The above proof and Proposition 2 show the following:

PROPOSITION 3. With the same assumptions as in Theorem 2 , let $f$ be the E-mapping of $D$ such that $f(1)=p, f^{\prime}(1)=v$ and $\operatorname{Im}\left\langle f^{\prime \prime}(1), \bar{\nu}(p)\right\rangle=0$. Let $g$ be an analytic disc from $U$ into $D, C^{3}$ on $\bar{U}$ with $g(1)=p, g^{\prime}(1)=v$ and $\operatorname{Im}\left\langle g^{\prime \prime}(1), \bar{\nu}(p)\right\rangle=0$. If $g$ is not an E-mapping then we have either

$$
\left\langle f^{\prime \prime}(1), \bar{\nu}(p)\right\rangle<\left\langle g^{\prime \prime}(1), \bar{\nu}(p)\right\rangle,
$$

or

$$
\left\langle f^{\prime \prime}(1), \bar{\nu}(p)\right\rangle=\left\langle g^{\prime \prime}(1), \bar{\nu}(p)\right\rangle
$$

and

$$
\operatorname{Re}\left\langle f^{\prime \prime \prime}(1), \bar{\nu}(p)\right\rangle>\operatorname{Re}\left\langle g^{\prime \prime \prime}(1), \bar{\nu}(p)\right\rangle .
$$

So Proposition 3 gives the extremal property of $E$-mappings with respect to $p, v$.

\section{Hölder estimates and the existence part of Theorem 1. Let}

$\mathbf{F}=\{f \mid f: E$-mappings of $D$ satisfying $d(f(0), \partial D) \geq d(f(\varsigma), \partial D)$ for all $\varsigma \in \bar{U}\}$.

We have used $d(z, K)$ to denote the distance between the point $z$ and the set $K$. The family $\mathbf{F}$ is the set of $E$-mappings with the right parametrization as seen by the following:

LEMMA 7. Suppose that $\partial D \in C^{k}, k \geq 3$. There is a positive constant $C=$ $C(D)$ such that for every $f \in \mathbf{F}$ we have

$$
d(f(\varsigma), \partial D) \leq C(1-|\zeta|)^{1 / 2} \text { for all } \varsigma \in \bar{U} .
$$

ProOF. Since $\partial D$ is of class $C^{k}, k \geq 3$, there exists a positive constant $\mu$ such that for every boundary point $p$ of $D$ there is an open ball $B_{\mu}(p)$ of radius $\mu$ in $D$ with $\overline{B_{\mu}(p)} \cap \partial D=\{p\}$. For $f \in \mathbf{F}$, suppose $d(f(0), \partial D) \geq \mu$, (4) follows from [7]. In fact, it is proved that there exists a constant $C=C(D)$ such that $d(f(\varsigma), \partial D) \leq C(1-|\zeta|)$ in this case. So we assume that $d(f(0), \partial D)<\mu$. For $\varsigma \in U$ fixed, let $z_{1}, z_{2} \in \partial D$ satisfy $d(f(0), \partial D)=\left|f(0)-z_{1}\right|, d(f(\varsigma), \partial D)=\left|f(\varsigma)-z_{2}\right|$ respectively. Let $q_{1}, q_{2}$ be the centers of the $\mu$-balls $B_{\mu}\left(z_{1}\right), B_{\mu}\left(z_{2}\right)$ respectively. 
Denote by $\delta_{K}(p, q)$ the Kobayashi distance between two points $p, q$ in the set $K$. We have by the triangle inequality

$$
\begin{aligned}
\delta_{D}(f(0), f(\varsigma)) & \leq \delta_{D}\left(f(0), q_{1}\right)+\delta_{D}\left(q_{1}, q_{2}\right)+\delta_{D}\left(q_{2}, f(\varsigma)\right) \\
& \leq \delta_{B_{\mu}\left(z_{1}\right)}\left(f(0), q_{1}\right)+\delta_{D}\left(q_{1}, q_{2}\right)+\delta_{B_{\mu}\left(z_{2}\right)}\left(q_{2}, f(\varsigma)\right) .
\end{aligned}
$$

Let $C_{1}=\sup \delta_{D}\left(q, q^{\prime}\right)$ where $q, q^{\prime} \in D$ and $d(q, \partial D)=d\left(q^{\prime}, \partial D\right)=\mu$. Since $f$ is an $E$-mapping we have from the above inequality

$$
\frac{1}{2} \log \frac{1+|\zeta|}{1-|\zeta|} \leq \frac{1}{2} \log \frac{\mu+\left|q_{1}-f(0)\right|}{\mu-\left|q_{1}-f(0)\right|}+C_{1}+\frac{1}{2} \log \frac{\mu+\left|q_{2}-f(0)\right|}{\mu-\left|q_{2}-f(0)\right|}
$$

Because $\mu-\left|q_{1}-f(0)\right|=d(f(0), \partial D)$ and $\mu-\left|q_{2}-f(\varsigma)\right|=d(f(\varsigma), \partial D)$ we have

$$
d(f(0), \partial D) d(f(\varsigma), \partial D) \leq C_{2}(1-|\zeta|),
$$

where $C_{2}$ depends only on $\mu$ and $C_{1}$. Thus for $f \in \mathbf{F}$ we have $d(f(\varsigma), \partial D) \leq$ $C(1-|\zeta|)^{1 / 2}$ for all $\zeta \in \bar{U}$.

LEMMA 8. Let $D$ be as in Lemma 7. We can find a positive constant $C=C(D)$ such that when $f \in \mathbf{F}$

$$
\left|f\left(\varsigma_{1}\right)-f\left(\varsigma_{2}\right)\right| \leq\left. C\right|_{\zeta_{1}}-\left.\varsigma_{2}\right|^{1 / 4} \quad \text { for all } \varsigma_{1}, \varsigma_{2} \in \bar{U} .
$$

PROOF. By Lempert [7, p. 351] we have

$$
\left|f^{\prime}(\varsigma)\right| \leq C \frac{d(f(\varsigma), \partial D)^{1 / 2}}{(1-|\varsigma|)} \text { for all } \varsigma \in \bar{U}, C=C(D) \text {. }
$$

By (4) we obtain

$$
\left|f^{\prime}(\varsigma)\right| \leq C(1-|\zeta|)^{-3 / 4}
$$

The lemma then follows from the Hardy-Littlewood theorem.

The next proposition will be used in proving Theorems 1 and 2. For an analytic disc $f$ from $U$ into $D, d(f)$ denotes the diameter of the set $f(\bar{U})$.

Proposition 4. Assume that $\partial D \in C^{k}, k \geq 3$. Let $A$ be a family of $E$ mappings of $D$. Suppose there is a positive constant $C$ such that for all $f \in A$, $d(f) \geq C$. Then there is a compact set $K \subset D$ such that $f(U) \cap K \neq \varnothing$ for all $f \in A$.

ProOF. With new parameters we may assume that $A \subset \mathbf{F}$. Suppose there is no such compact subset $K$ in $D$. Write $D=\bigcup_{j=1} K_{j}$ where $K_{1} \subset K_{2} \subset \cdots \subset$ $K_{j} \subset \cdots$ are compact subsets of $D$. Then for each $j$ we can find $f_{j} \in A$ such that $f_{j}(\bar{U}) \cap K_{j}=\varnothing$. Since $A \subset \mathbf{F}$ we can find a subsequence of $\left\{f_{j}\right\}$ which converges uniformly on $\bar{U}$ by (5). Let $f$ be its limit. We have $d(f) \geq C$, f holomorphic in $U$ and $f(\bar{U}) \cap K_{j}=\varnothing$ for $j=1,2, \ldots$. Hence we must have $f(U) \subset \partial D$. This is impossible, for $D$ is strictly linearly convex. Therefore the proposition is true.

Now we can finish the proof of Theorem 1.

Existence part of Theorem 1. When both $p, q$ are in $D$ Lempert [7] has proved the existence of an $E$-mapping $f$ passing through them. We shall use his result to prove the case when both $p, q$ are on $\partial D$; the case $p \in \partial D, q \in D$ can be proved similarly.

Let $\left\{p_{j}\right\},\left\{q_{j}\right\}$ be two sequences of points in $D$ such that $p_{j} \rightarrow p, q_{j} \rightarrow q$ as $j \rightarrow \infty$. From [7], there is for each $j$ an $E$-mapping $f_{j}$ of $D$ passing through 
$p_{j}, q_{j}$. Since $d\left(f_{j}\right) \geq\left|p_{j}-q_{j}\right|$, we may assume that there is a positive constant $C$ such that $d\left(f_{j}\right) \geq C$ for every $j$. Reparametrizing $f_{j}$ and by virtue of Proposition 4 we may assume that $\left\{f_{j}(0)\right\}$ is contained in a compact subset $K$ in $D$. Thus by Propositions 8,10 of $[7]$ both $\left\{f_{j}\right\}$ and $\left\{\tilde{f}_{j}\right\}$ satisfy a uniform $\frac{1}{2}$-Hölder estimate on $\bar{U}$. Standard normal family argument then shows that there is a subsequence of $\left\{f_{j}\right\}\left(\left\{\tilde{f}_{j}\right\}\right.$ resp.) that converges uniformly to a map $f_{0}\left(\tilde{f}_{0}\right.$ resp.) holomorphic in $U$ and $\frac{1}{2}$-Hölder continuous on $\bar{U}$. It is easy to see, by Theorem 2 of [7], that $f_{0}$ is an $E$-mapping of $D$. Obviously $f_{0}$ passes through $p$ and $q$.

3. Existence part of Theorem 2. Let $p$ be a boundary point of $D, \sqrt{-1} v \in$ $T_{p}(\partial D)$ with $\langle v, \bar{\nu}(p)\rangle>0$. To prove the existence of an $E$-mapping with given $p, v$ we first use Vitushkin's normal form to write $\partial D$ near $p$ in a form more convenient to analyze. In fact, it suffices to use the partial normal form which does not require the trace conditions for the normal form.

LEMMA 9. Let $M$ be a $C^{k}, k \geq 3$, strongly pseudoconvex hypersurface in $\mathbf{C}^{n}$. Let $p \in M$ and $\sqrt{-1} v \in T_{p}(M) \backslash\left\{\right.$ maximal complex subspace of $\left.T_{p}(M)\right\}$. Then there is a biholomorphic map $\varphi$ defined in a neighborhood $V$ of $p$ in $\mathbf{C}^{n}$ such that $\varphi(p)=(1,0, \ldots, 0), \varphi_{*}(p) v=(\lambda, 0, \ldots, 0), \lambda \in \mathbf{R}^{+}$, and $\varphi(V \cap M)$ is defined by the function

$$
\begin{aligned}
1-\left|z_{1}\right|^{2} & =\left|z^{\prime}\right|^{2}+\sum_{|\alpha|,|\beta| \geq 2} a_{\alpha \beta}\left(\arg z_{1}\right) z^{\prime \alpha} \bar{z}^{\prime \beta}+R(z) \\
& =\left|z^{\prime}\right|^{2}+A(z)+R(z),
\end{aligned}
$$

where $z^{\prime}=\left(z_{2}, \ldots, z_{n}\right), \alpha, \beta \in \mathbf{N}^{n-1}$, are multi-indices, $a_{\alpha \beta}$ is real analytic for every $\alpha, \beta, \bar{a}_{\beta \alpha}=a_{\alpha \beta}$ and $R(z)=O\left(|z-(1,0, \ldots, 0)|^{k}\right)$ when $k<\infty, R(z)=$ $O\left(|z-(1,0, \ldots, 0)|^{N}\right)$ for any $N \in \mathbf{N}, N \geq 3$ when $k=\infty$, and $R(z)=0$ when $k=\omega$.

PROOF. When $k=\omega(6)$ is nothing but the partial normal form of Vitushkin. We prove the case $k<\infty$; the case $k=\infty$ follows easily. Suppose that, in a neighborhood of $p, M$ is defined by the $C^{k}$ function $\rho(z)=0$. We may assume that $p$ is the origin in $\mathbf{C}^{n}$. Taylor's formula at $p$ gives

$$
\rho(z)=\sum_{|\alpha+\beta| \leq k-1} C_{\alpha \beta} z^{\alpha} \bar{z}^{\beta}+r(z)=a(z)+r(z),
$$

where $\alpha, \beta \in \mathbf{N}^{n}, C_{\alpha, \beta} \in \mathbf{C}, \bar{C}_{\alpha \beta}=C_{\beta \alpha}$ and $r(z)=O\left(|z|^{k}\right)$. Since $M$ is strongly pseudoconvex, so is $a(z)$ if $|z|$ is small. There is a biholomorphic map $\varphi$ defined in a neighborhood $\tilde{V}$ of $p$ such that $\{z \mid a(z)=0\}$ is transformed to

$$
1-\left|z_{1}\right|^{2}=\left|z^{\prime}\right|^{2}+\sum_{|\alpha|,|\beta| \geq 2} a_{\alpha \beta}\left(\arg z_{1}\right) z^{\prime \alpha} \bar{z}^{\prime \beta}
$$

with $\varphi(0)=(1,0, \ldots, 0), \varphi_{*}(0) v=(\lambda, 0, \ldots, 0), \lambda \in \mathbf{R}^{+}, \bar{a}_{\alpha \beta}=a_{\beta \alpha}$ and $a_{\alpha \beta}$ real analytic for all $\alpha, \beta$. Since $r(z)=O\left(|z|^{k}\right)$ the biholomorphic map $\varphi$ sends $\{z \mid \rho(z)=0\}$ in a neighborhood $V$ of $(0, \ldots, 0)$ to the form $(6)$.

The next proposition concerning the perturbation of domains is needed in proving both Theorems 2, 3. Since its proof is long and parallel to Proposition 10 of [6] we postpone its proof till the next section. 
PROPOSITION 5. Let $D_{t}, t \in[0,1]$, be a family of bounded strictly linearly convex domains with $C^{k}$ boundaries, which is $C^{k}$ dependent on $t, k \geq 6$. Assume that there is a fixed point $p \in \partial D_{t}$ for all $t \in[0,1]$, and both the tangent spaces $T_{p}\left(\partial D_{t}\right)$ and the complex Hessians $H_{t}(p)$ of $\partial D_{t}$ at $p$ are independent of $t \in[0,1]$. If for a given iv $\in T_{p}\left(\partial D_{t}\right)$ there exists an E-mapping $f_{0}$ of $D_{0}$ such that $f_{0}(1)=p$, $f_{0}^{\prime}(1)=v$ and $\operatorname{Im}\left\langle f_{0}^{\prime \prime}(1), \bar{\nu}(p)\right\rangle=0$, then there is a $t_{0} \in(0,1]$ such that for each $t \in\left[0, t_{0}\right]$ there is an E-mapping $f_{t}$ of $D_{t}$ satisfying $f_{t}(1)=p, f_{t}^{\prime}(1)=v$ and $\operatorname{Im}\left\langle f_{t}^{\prime \prime}(1), \bar{\nu}(p)\right\rangle=0$. The map $t \rightarrow f_{t}$ is of class $C^{k-4}$.

Assuming this proposition we prove the

Existence part of Theorem 2. Using Lemma 9 we write $\partial D$ near $p$ in the form (6). When $k=\infty$ we take $N \geq 14$ for $R(z)$. Let $\psi(x) \in C_{0}^{\infty}(\mathbf{R})$ with $0 \leq \psi \leq 1$ and

$$
\psi(x)= \begin{cases}1 & \text { if }|x|<\varepsilon / 4, \\ 0 & \text { if }|x|>\varepsilon / 2\end{cases}
$$

where $\varepsilon$ is chosen so that

(i) the function $A(z)$ and $R(z)$ in $(6)$ are defined in $|z-(1,0, \ldots, 0)|<\varepsilon$;

(ii) for $t \in(0,1]$ we define $\psi_{t}(x)=\psi\left(t^{-1} x\right)$ and define domains

$$
\begin{aligned}
D_{t}=\left\{\left.z \in \mathbf{C}^{n}|1-| z_{1}\right|^{2}>\left|z^{\prime}\right|^{2}+\psi(|z-(1,0, \ldots, 0)|) A(z)\right. & \\
& \left.+\psi_{t}(|z-(1,0, \ldots, 0)|) R(z)\right\} .
\end{aligned}
$$

Also we define $\psi_{0}(x)=0$ and define

$$
D_{0}=\left\{\left.z \in \mathbf{C}^{n}|1-| z_{1}\right|^{2}>\left|z^{\prime}\right|^{2}+\psi(|z-(1,0, \ldots, 0)|) A(z)\right\} .
$$

We choose $\varepsilon$ so small that the domains $D_{t}$ are strictly linearly convex.

It is easy to see that for each $t \in(0,1]$ there is a neighborhood $W_{t} \subset V$ of $p$ in $\mathrm{C}^{n}$ such that $\varphi\left(W_{t} \cap D\right) \subset D_{t}$ and $\varphi\left(W_{t} \cap \partial D\right)$ is a neighborhood of $(1,0, \ldots, 0)$ on $\partial D_{t}$. For $t=0$ we observe that

The map $f_{0}: \bar{U} \rightarrow \bar{D}_{0}$ defined by $f_{0}(\varsigma)=(\varsigma, 0, \ldots, 0)$ is an $E$ mapping of $D_{0}$ satisfying $f_{0}(1)=(1,0, \ldots, 0), f_{0}^{\prime}(1)=(1, \ldots, 0)$ and $\operatorname{Im}\left\langle f_{0}^{\prime \prime}(1),(1, \ldots, 0)\right\rangle=0$.

Noting that $\partial D \in C^{k}, k \geq 14$, we see, by the definitions of $D_{t}, t \in[0,1]$, that the assumptions of Proposition 5 are satisfied. Hence for some $t_{0} \in(0,1]$ we have $E$-mappings $f_{t}$ of $D_{t}$ satisfying $f_{t}(1)=(1,0, \ldots, 0), f_{t}^{\prime}(1)=(1,0, \ldots, 0)$ and $\operatorname{Im}\left\langle f_{t}^{\prime \prime}(1),(1,0, \ldots, 0)\right\rangle=0$ for each $t \in\left[0, t_{0}\right]$. Note when $k=\omega$ we have $D_{t}=D_{0}$ for all $t \in(0,1]$ and we may take $t_{0}=1$. Let $\left\{s_{j}\right\}$ be a sequence in $(0,1)$ converging to 1 as $j \rightarrow \infty$ and $\left\{f_{t_{0}}\left(s_{j}\right)\right\} \subset \varphi\left(W_{t_{0}} \cap D\right)$. Let $p_{j}=\varphi^{-1}\left(f_{t_{0}}\left(s_{j}\right)\right)$ and $v_{j}=\varphi^{*}\left(f_{t_{0}}^{\prime}\left(s_{j}\right)\right)$. From [7], for each $j$ we can find a unique $E$-mapping $f_{j}$ of $D$ with $f_{j}\left(s_{j}\right)=p_{j}, f_{j}^{\prime}\left(s_{j}\right)=\mu_{j} v_{j}, \mu_{j} \in \mathbf{R}^{+}$. We claim that there is a positive constant $c$ such that $d\left(f_{j}\right) \geq c$ for all $j=1,2, \ldots$ For, if not, we can find a subsequence of $\left\{f_{j}\right\}$, still denoted by $\left\{f_{j}\right\}$, such that $d\left(f_{j}\right) \rightarrow 0$ as $j \rightarrow \infty$. Thus for some $N \in \mathbf{N}$ we have $f_{j}(\bar{U}) \subset W_{t_{0}} \cap \bar{D}$ when $j \geq N$. Since $\varphi$ is a biholomorphic map on $W_{t_{0}}$, it can be easily seen that $\varphi \circ f_{j}$ is an $E$-mapping of $D_{t_{0}}$ when $j \geq N$. By our construction we have $\varphi \circ f_{j}\left(s_{j}\right)=f_{t_{0}}\left(s_{j}\right)$ and $\left(\varphi \circ f_{j}\right)^{\prime}\left(s_{j}\right)=\mu_{j} f_{t_{0}}^{\prime}\left(s_{j}\right)$. Since both $\varphi \circ f_{j}$ and $f_{t_{0}}$ are $E$-mappings of $D_{t_{0}}$ we must have $\mu_{j}=1$ and $\varphi \circ f_{j} \equiv f_{t_{0}}$ when $j \geq N$. But since $d\left(f_{j}\right) \rightarrow 0$ as $j \rightarrow \infty$ we should have $d\left(\varphi \circ f_{j}\right) \rightarrow 0$ as $j \rightarrow \infty$. This is absurd, for $f_{t_{0}}$ is a fixed $E$-mapping of $D_{t_{0}}$. This proves the claim. 
By means of Proposition 4 we can reparametrize $f_{j}$, denoted again by $f_{j}$, such that $f_{j}(0)$ is contained in a compact subset of $D$ for all $j=1,2, \ldots$ We then argue exactly as in the proof of Theorem 1 that a subsequence of $\left\{f_{j}\right\}$ converges to a nondegenerate $E$-mapping $g$ of $D$. It is easy to check that $g$ passes through $p$ and its derivative at $p$ is a multiple of $v$. Now composing $g$ with a rotation of $U$ we may assume that $g(1)=p$. Then $g^{\prime}(1)$ is a positive multiple of $v$. By composing $g$ with an automorphism of $U$ we obtain the desired $E$-mapping.

4. Perturbation of domains. The proof of Proposition 5 is essentially the same as [6, Proposition 10, p. 446]. To avoid repetition we only sketch the part of the proof where they are identical. First we recall Proposition 9 of [6].

Proposition. Suppose that $D$ is strongly convex and $\partial D \in C^{k}, k \geq 4$. Let $f: \bar{U} \rightarrow \bar{D}$ be an E-mapping of $D$. Given $\varepsilon>0$ we can find a new coordinate $w$ in a neighborhood of $f(\bar{U})$ and $a C^{k}$ defining function $r$ of $D$ such that in this coordinate the distance between

(i) $f(\varsigma)$ and $(\varsigma, 0, \ldots, 0)$;

(ii) $r_{w}(f(\varsigma))$ and $(\bar{\zeta}, 0, \ldots, 0)$;

(iii) $r_{w \bar{w}}(f(\varsigma))$ and the $n \times n$ unit matrix $I_{n}$;

(iv) $r_{w_{1} w}(f(\varsigma))$ and $(0, \ldots, 0)$

are less than $\varepsilon$ for $\zeta \in \partial U$. Also for each $\zeta \in \partial U$ the norm of the matrix $\left(r_{w^{\prime} w^{\prime}}(f(s))\right)\left(w^{\prime}=\left(w_{2}, \ldots, w_{n}\right)\right)$ is less than $\sigma<1$, where $\sigma$ is independent of $\varepsilon$. For an $m \times m$ matrix $M$ the norm of $M$ is defined by $[M]=\sup \{\mid M v \| v \in$ $\left.\mathbf{C}^{m},|v|=1\right\}$.

REMARK 3. This proposition holds when $D$ is strictly linearly convex by exactly the same proof.

PROOF OF PROPOSITION 5. Without loss of generality we may assume that $\nu^{t}(p)=(1,0, \ldots, 0)$ for all $t \in[0,1]$ where $\nu^{t}=\left(\nu_{1}^{t}, \ldots, \nu_{n}^{t}\right)$ denotes the outward normal to $\partial D_{t}$ at $p$. Since only a neighborhood $V$ of $f_{0}(\bar{U})$ is concerned we may assume that we are dealing in a coordinate given by the above Proposition (cf. Remark 3). It follows that for $t$ small the map

$$
\partial D_{t} \cap V \ni z \rightarrow\left(z, \frac{\bar{\nu}_{2}^{t}(z)}{\bar{\nu}_{1}^{t}(z)}, \ldots, \frac{\bar{\nu}_{n}^{t}(z)}{\bar{\nu}_{1}^{t}(z)}\right) \in \mathbf{C}^{2 n-1}
$$

is well defined. Let $M_{t}$ be the image of $\partial D_{t} \cap V$ under this map. Since $D_{t}$ is strongly pseudoconvex, we see that for $t$ and $V$ small enough $\left\{M_{t}\right\}$ is a $C^{k-1}$ family of totally real submanifolds of $\mathbf{C}^{2 n-1}$, diffeomorphic to $\partial U \times \mathbf{R}^{2 n-2}$. Let $F_{t}$ be a $C^{k-1}$ diffeomorphism from $M_{t}$ to $M_{0}, C^{k-1}$ with respect to $t$.

For $w \in \mathbf{C}^{2 n-1}$, denote by $w_{a}$ the first $n$ components of $w$ and by $w_{d}$ the last $n-1$ components of $w$. It suffices to find a continuous family of $\frac{1}{2}$-Hölder continuous maps $\left\{g_{t}\right\}$ from $\bar{U}$ into $\mathbf{C}^{2 n-1}$, holomorphic in $U$ such that

$$
g_{0}=f_{*}=\left(f_{0}, \tilde{f}_{2} / \tilde{f}_{1}, \ldots, \tilde{f}_{n} / \tilde{f}_{1}\right),
$$

where $\tilde{f}=\left(\tilde{f}_{1}, \ldots, \tilde{f}_{n}\right)$ is the holomorphic map corresponding to the $E$-mapping $f_{0}$, and

$$
g_{t}(\partial U) \subset M_{t}
$$

Then $\left\{\left(g_{t}\right)_{a}\right\}$ is the desired family of $E$-mappings as shown in [6]. 
Let $X, Y$ be Banach spaces defined by

$$
\begin{aligned}
& X=\left\{u \mid u: \partial U \rightarrow \mathbf{C}^{2 n-1}, u \in C^{5 / 2}(\partial U)\right\}, \\
& Y=\left\{\left(u_{j k}\right)_{(2 n-1) \times(2 n-1)} \mid u_{j k}: \partial U \rightarrow \mathbf{C}, u_{j k} \in C^{5 / 2}(\partial U)\right\} .
\end{aligned}
$$

For a map $h: \mathbf{R} \rightarrow X$ or $Y$ we write $\dot{h}=\dot{h}_{t}=d h / d t$ and for a map $H: \mathbf{C}^{2 n-1} \rightarrow$ $\mathbf{C}^{2 m}$ we write $H^{\prime}=\partial H / \partial w, \bar{H}^{\prime}=\partial H / \partial \bar{w}$ where $w \in \mathbf{C}^{2 n-1}$.

Let $T_{1}, \ldots, T_{2 n-1}$ be everywhere linearly independent $C^{k-1}$ vector fields on $M_{0}$. Since $M_{0}$ is totally real, $T_{1}, \ldots, T_{2 n-1}$ are linearly independent over $\mathbf{C}$. It follows that the matrix $T \in \mathbf{C}^{2 n-1} \times \mathbf{C}^{2 n-1}$ with $T_{j}, j=1, \ldots, 2 n-1$, as column vectors is invertible.

For a map $g_{t}$ satisfying (8), (9), $\left.g_{t}\right|_{\partial U}$ can be considered as an element of $X$ (in fact $g_{t} \in C^{1 / 2}(\bar{U})$ implies that $g_{t} \in C^{k-2}(\bar{U})$, see $\left.[\mathbf{6}, \S 5]\right)$. From

$$
\begin{aligned}
\frac{d}{d t}\left(F_{t} \circ g_{t}\right) & =\dot{F}_{t} \circ g_{t}+\left(F_{t}^{\prime} \circ g_{t}\right) \dot{g}_{t}+\left(\bar{F}_{t}^{\prime} \circ g_{t}\right) \dot{\bar{g}}_{t} \\
& =\sum_{j=1}^{2 n-1} \gamma_{j}\left(T_{j} \circ F_{t} \circ g_{t}\right)=\left(T \circ F_{t} \circ g_{t}\right) \gamma
\end{aligned}
$$

where $\gamma_{j} \in \mathbf{R}$, we obtain

$$
\begin{aligned}
0=\operatorname{Im}\{ & \left(T^{-1} \circ F_{t} \circ g_{t}\right)\left(\dot{F}_{t} \circ g_{t}\right) \\
+ & {\left.\left[\left(T^{-1} \circ F_{t} \circ g_{t}\right)\left(F_{t}^{\prime} \circ g_{t}\right)-\overline{\left(T^{-1} \circ F_{t} \circ g_{t}\right)}\left(\bar{F}_{t}^{\prime} \circ g_{t}\right)\right] \dot{g}_{t}\right\} . }
\end{aligned}
$$

For $(t, x)$ in a neighborhood of $\left(0, f_{*}\right)$ we write

$$
B(t, x)=\left(T^{-1} \circ F_{t} \circ x\right)\left(\dot{F}_{t} \circ x\right): \mathbf{R} \times X \rightarrow X
$$

and

$$
A(t, x)=\left(T^{-1} \circ F_{t} \circ x\right)\left(F_{t}^{\prime} \circ x\right)-\overline{\left(T^{-1} \circ F_{t} \circ x\right)}\left(\bar{F}_{t}^{\prime} \circ x\right): \mathbf{R} \times X \rightarrow Y .
$$

We can write (10) as

$$
\operatorname{Im}\left\{A\left(t, g_{t}\right) \dot{g}_{t}+B\left(t, g_{t}\right)\right\}=0 .
$$

Note that $A\left(0, f_{*}\right)$ is invertible; in fact $A^{-1}\left(0, f_{*}\right)=T \circ f_{*}$. Let

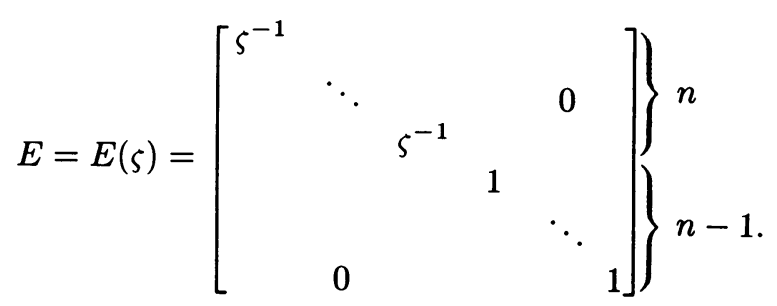

Following [6, Proposition 10], for $(t, x)$ close to $\left(0, f_{*}\right)$ there exist $R(t, x): \partial U \rightarrow$ $\mathrm{GL}(2 n-1, \mathbf{R})$ and $H(t, x): \bar{U} \rightarrow \mathrm{GL}(2 n-1, \mathbf{C})$ of class $C^{5 / 2}, H$ holomorphic in $U$ and $R(t, x)(1)=I_{2 n-1}$ such that

$$
E A^{-1}(t, x) R(t, x)=H(t, x) \quad \text { on } \partial U
$$

and the map $(t, x) \rightarrow H(t, x)$ is of class $C^{k-5}$. 
Since we are looking for $g_{t}$ with $\left(g_{t}\right)_{a}(1)=p,\left(g_{t}^{\prime}\right)_{a}(1)=v$ and $\operatorname{Im}\left\langle\left(g_{t}^{\prime \prime}\right)_{a}(1), \bar{\nu}(p)\right\rangle$ $=0$ for $t$ small, we must have $\left(\dot{g}_{t}\right)_{a}(1)=0$ and $\left(\dot{g}_{t}^{\prime}\right)_{a}(1)=0$ for $t$ small. On the other hand, $\nu^{t}(p)$ and $H_{t}(p)$ are, by assumption, independent of $t$. We therefore have that $\dot{g}_{t}(1)=0$ and $\dot{g}_{t}(1)=0$. From these remarks and the fact that $\zeta(\varsigma-1)^{-2}$ is real for $\varsigma \in \partial U \backslash\{1\}$ we obtain from (11), (12)

$$
\operatorname{Im} H^{-1}\left(t, g_{t}\right) E_{\zeta}(\varsigma-1)^{-2} \dot{g}_{t}=\beta\left(t, g_{t}\right) \in C^{1 / 2}(\partial U),
$$

where $\beta\left(t, g_{t}\right)=-\operatorname{Im} R^{-1}(t, g) \varsigma(\zeta-1)^{-2} B\left(t, g_{t}\right)$. Fix $(t, x)$, complete $i \beta(t, x)$ to a map $\gamma(t, x)$ holomorphic in $U$ such that $\operatorname{Re} \gamma(t, x)(1)=0$ and $\operatorname{Im} \gamma(t, x)(\varsigma)=$ $\beta(t, x)(\varsigma), \varsigma \in \partial U$. By the result on Hilbert transforms we know that $\gamma$ is of class $C^{k-5}$ in a neighborhood of $\left(0, f_{*}\right)$ in $\mathbf{R} \times X$. Hence (13) can be written in the form

$$
H^{-1}\left(t, g_{t}\right) E_{\zeta}(\varsigma-1)^{-2} \dot{g}_{t}=\gamma\left(t, g_{t}\right)+u(t)
$$

where $u: \mathbf{R} \rightarrow \mathbf{R}^{2 n-1}$ is to be determined by the next lemma which will be proved at the end of this section.

LEMMA 10. For $\varsigma_{0} \in \bar{U},(t, x)$ fixed we define the maps

$$
\begin{gathered}
\psi_{a}\left(\varsigma_{0}\right): \mathbf{R}^{2 n-1} \ni u \rightarrow\left(H(t, x)\left(\varsigma_{0}\right) u\right)_{a} \in \mathbf{C}^{n} \approx \mathbf{R}^{2 n}, \\
\psi_{d}\left(\varsigma_{0}\right): \mathbf{R}^{2 n-1} \ni u \rightarrow\left(H(t, x)\left(\varsigma_{0}\right) u\right)_{d} \in \mathbf{C}^{n-1} \approx \mathbf{R}^{2 n-2} .
\end{gathered}
$$

Then for $(t, x)$ close to $\left(0, f_{*}\right)$ the following system of equations

$$
\left\{\begin{array}{l}
(H(t, x) \gamma(t, x))_{d}(0)+\psi_{d}(0) u=0, \\
\operatorname{Im}\left\langle(H(t, x) \gamma(t, x))_{a}(1)+\psi_{a}(1) u, \bar{\nu}(p)\right\rangle=0
\end{array}\right.
$$

has a unique solution $u$.

With this $u$ we solve the ordinary differential equations

$$
\left\{\begin{array}{l}
\dot{g}_{t}=(\varsigma-1)^{2} \varsigma^{-1} E^{-1} H\left(t, g_{t}\right)\left(\gamma\left(t, g_{t}\right)+u\left(t, g_{t}\right)\right), \\
g_{0}=f_{*}
\end{array}\right.
$$

such that $\left(g_{t}\right)_{a}=f_{t}$ is a family of $E$-mappings from $\bar{U}$ into $\bar{D}_{t}$ with $f_{t}(1)=p$, $f_{t}^{\prime}(1)=v$ and $\operatorname{Im}\left\langle f_{t}^{\prime \prime}(1), \bar{\nu}(p)\right\rangle=0$. Note that the right-hand side of the first equation in (16) does not involve $g^{\prime}(1), g^{\prime \prime}(1)$ any more and is $C^{k-5}$ with respect to $(t, x)$, holomorphic with respect to $\varsigma \in U$. Hence (16) has the unique $C^{k-4}$ solution $g_{t}$ such that for $f_{t}=\left(g_{t}\right)_{a}$ we have $f_{t}(1)=p, f_{t}^{\prime}(1)=v$ and $\operatorname{Im}\left\langle\dot{f}_{t}^{\prime \prime}(1), \bar{\nu}(p)\right\rangle=0$. It follows that $\operatorname{Im}\left\langle f_{t}^{\prime \prime}(1), \bar{\nu}(p)\right\rangle$ is a constant independent of $t$. But when $t=0$ it equals 0 by assumption. This concludes the proof.

PROOF OF LEMMA 10. The proof goes under the same assumptions as that of Proposition 5. We claim:

(i) for any $\varsigma_{0} \in \bar{U}, \psi_{a}\left(\varsigma_{0}\right)$ is one-one and its image is transversal to $\nu(p)$;

(ii) for any $\varsigma_{0} \in \bar{U}$, the kernel of $\psi_{d}\left(\varsigma_{0}\right)$ is one dimensional and if $u \neq 0$ is such that $\psi_{d}\left(\varsigma_{0}\right) u=0$, then $\psi_{a}\left(\varsigma_{0}\right) u=\left(s_{1}, \ldots, s_{n}\right), s_{1} \notin \mathbf{R}$.

The proof of (i) can be found in [6, p. 454]. In fact, it is also proved that if $H(t, x)\left(\varsigma_{0}\right) u=\left(s_{1}, \ldots, s_{n}, 0, \ldots, 0\right)$ then $s_{1}$ cannot be real. It remains to prove the first part of (ii). Suppose $u_{1}, u_{2}$ are two independent vectors in $\mathbf{R}^{2 n-1}$ such that $\psi_{d}\left(\varsigma_{0}\right) u_{1}=\psi_{d}\left(\varsigma_{0}\right) u_{2}=0$. We can easily construct from $u_{1}$ and $u_{2}$ a vector $u \neq 0$ such that $\psi_{d}\left(\varsigma_{0}\right) u=0$ and $\psi_{a}\left(\varsigma_{0}\right) u=\left(t_{1}, \ldots, t_{n}\right)$ with $t_{1} \in \mathbf{R}$. This contradicts the second part of (ii). 
It then follows from the above that if $0 \neq u \in \mathbf{R}^{2 n-1}$ is such that $H(t, x)\left(\varsigma_{0}\right) u=$ $\left(s_{1}, \ldots, s_{2 n-1}\right), s_{1} \in \mathbf{R}$, we must have $\left(s_{2}, \ldots, s_{n}\right) \neq 0$ and $\left(s_{n+1}, \ldots, s_{2 n-1}\right) \neq 0$. Also, if we let $W=\left\{u \in \mathbf{R}^{2 n-1} \mid\right.$ the first component $\left(\psi_{a}\left(\varsigma_{0}\right) u\right)_{1}$ of $\psi_{a}\left(\varsigma_{0}\right) u$ is real $\}$, it is easy to see from the proof of (i), (ii) that $\psi_{d}\left(\varsigma_{0}\right)$ is $1-1$ on $W$ and $\psi_{d}\left(\varsigma_{0}\right) W=$ $\psi_{d}\left(\varsigma_{0}\right) \mathbf{R}^{2 n-1}$. Therefore $W$ is of real $(2 n-2)$ dimension and so is the space of the solutions of the second equation of (15). On the other hand, the solutions to the first equation of (15) are of real one dimension and can be expressed as $u=v+s w$ where $s \in \mathbf{R}$ and $v, w$ are fixed vectors in $\mathbf{R}^{2 n-1}$ satisfying $\psi_{d}(0) w=0$ and $\left(\psi_{a}(0) v\right)_{1} \in \mathbf{R}$; note that this expression is unique once $v, w$ are fixed. From (ii), it follows that $\psi_{a}(0) w \notin \mathbf{R}$. By explicit calculations, when $D=B_{n}, g(\varsigma)=(\varsigma, 0, \ldots, 0), H(0, g)(\varsigma)$ is a constant matrix independent of $\zeta \in \bar{U}$ (see [6, p. 453]). Therefore in this case, $\psi_{a}(0) w \notin \mathbf{R}$ implies that $\psi_{a}(1) w \notin \mathbf{R}$. For general $D$, by Remark 3 which we have already applied at the beginning of the proof of Proposition 5, the coordinate we choose in a neighborhood of $f_{0}$ can be made as close to the ball as we want. Hence the first row of $H\left(0, f_{*}\right)$ is a small perturbation of the first row of $H(0, g)$ (cf. [6, p. 454]) and we have $\psi_{a}(1) w \notin \mathbf{R}$ also. For $(t, x)$ close enough to $\left(0, f_{*}\right)$, by the continuity of $H$, the same assertion also holds. Hence, we can choose a unique $s \in \mathbf{R}$ such that $u=v+s w$ is the solution to the second equation of (15). Therefore (15) can be solved uniquely for $(t, x)$ close to $\left(0, f_{*}\right)$.

REMARK 4. With the help of Theorem 2 the conclusion of Proposition 5 can be strengthened to $t_{0}=1$.

5. Proof of Theorem 3. It is easy to construct the map $\Phi$ from Theorems 1 and 2. But to prove the regularity, we need the following analogue of Proposition 5 .

PROPOSITION $5^{\prime}$. Let $\left\{D_{t}\right\}, t \in[0,1]$, be a family of bounded strictly linearly convex domains in $\mathbf{C}^{n}$ with $C^{k}$ boundaries, $k \geq 14$, and $C^{r}$ dependent on $t, r \geq 6$. Assume that there is a point $p \in \partial D_{t}$ for all $t \in[0,1]$ and both the tangent spaces $T_{p}\left(\partial D_{t}\right)$ and the complex Hessians $H_{t}(p)$ of $\partial D_{t}$ at $p$ are independent of $t$. Also assume that $\left\{z_{t}\right\}$ is a $C^{k}$ curve in $\mathbf{C}^{n}$ with $z_{t} \in \bar{D}_{t} \backslash\{p\}, t \in[0,1]$. If for $t=0$, there is an E-mapping $f_{0}$ of $D_{0}$ passing through $p$ and $z_{0}$ satisfying $f_{0}(1)=p$, $f_{0}\left(\xi_{0}\right)=z_{0}, \xi_{0} \in[-1,1)$, then for all $t \in(0,1]$ there exist E-mappings $f_{t}$ of $D_{t}$ with $f_{0}(1)=p, f_{t}\left(\xi_{t}\right)=z_{t}, \xi_{t} \in[-1,1)$ and $\left\langle f_{t}^{\prime}(1), \bar{\nu}(p)\right\rangle=\left\langle f_{0}^{\prime}(1), \bar{\nu}(p)\right\rangle$. Furthermore, the maps $t \rightarrow f_{t}, t \rightarrow \xi_{t}$ are of class $C^{k-4}$.

Since the proof is almost the same as that of Proposition 5, we only give a brief sketch of it. Details can be found in [6, pp. 446-455].

Sketch of the proof. The existence of $f_{t}$ follows from [6, Theorem 2] or [7, Theorem 3] if $z_{t} \in D_{t}$ and from Theorem 1 if $z_{t} \in \partial D_{t}$.

It remains to prove the regularity of $f_{t}$ and $\xi_{t}$ with respect to $t$. We may assume that $t$ is close to 0 and proceed as in the proof of Proposition 5. The difference is that here we require that $\left(g_{t}\right)_{a}(1)=p$ and the first component of $g_{t}^{\prime}(1)$ always equals 1 . The matrix $E$ is then replaced by

$$
\tilde{E}=\tilde{E}(\varsigma)=\left[\begin{array}{cccc}
(\varsigma-1)^{-2} & & & 0 \\
& (\varsigma-1)^{-1} & & \\
0 & & \ddots & (\varsigma-1)^{-1}
\end{array}\right]
$$


and the factor $\varsigma(\varsigma-1)^{-2}$ in (13) is no longer needed. We now solve the system of ordinary equations

$$
\left\{\begin{array}{l}
\dot{g}_{t}=\tilde{E}^{-1} H\left(t, g_{t}\right)\left(\gamma\left(t, g_{t}\right)+u\left(t, g_{t}\right)\right) \\
g_{0}=f_{*}
\end{array}\right.
$$

where $u\left(t, g_{t}\right)$ is determined exactly as in [6, p. 451]. Since $f_{t}=\left(g_{t}\right)_{a}$ is the solution of $(16)^{\prime}$, the regularity of $f_{t}, \xi_{t}$ with respect to $t$ then follows from equation $(16)^{\prime}$. Note that $f_{t}^{\prime}(1)$ is of class $C^{k-5}$ with respect to $t$.

We now define the map $\Phi$. First, we observe that the map $\tau: \partial D \rightarrow \partial B_{n}$ defined by $\tau(p)=\nu(p) /|\nu(p)|$ is a $C^{k-1}$ surjective map, and it is injective if $D$ is strictly convex. For $p \in \partial D$, let $L_{p}=\left\{v=\langle w, \bar{\nu}(p) /|\nu(p)|\rangle w\left|\sqrt{-1} w \in T_{p}(\partial D),\right| w \mid=1\right.$ and $\langle w, \nu(p)\rangle>0\}$. Then $L_{p}$ is a $C^{k-1}$ submanifold of $T_{p}(\partial D)$ of real dimension $2 n-2$. For $v \in L_{p}$, denote by $f(p, v, \zeta)$ the $E$-mapping of $D$ parametrized by the conditions $f(p, v, 1)=p, f^{\prime}(p, v, 1)=v$ and $\operatorname{Im}\left\langle f^{\prime \prime}(p, v, 1), \bar{\nu}(p)\right\rangle=0$ where " $"$ denotes differentiation with respect to $\varsigma$. The map $\Phi$ is then defined as follows:

$$
\Phi(p, q)=\left\{\begin{array}{cl}
\nu(p) /|\nu(p)| & \text { if } q \in \bar{D} \backslash\{p\}, \text { where } v_{p}(q) \in L_{p}, \zeta_{p}(q) \in \bar{U} \\
+\left(\zeta_{p}(q)-1\right) v_{p}(q) & \text { are such that } f\left(p, v_{p}(q), \cdot\right) \text { is the normalized } \\
& E \text {-mapping passing through } p, q \text { and } \\
& f\left(p, v_{p}(q), \zeta_{p}(q)\right)=q, \\
\nu(p) /|\nu(p)| & \text { if } q=p .
\end{array}\right.
$$

The existence of $f\left(p, v_{p}(q), \cdot\right)$ is guaranteed by Theorems 1 and 2 . It is easy to see that for fixed $p \in \partial D$, the map $\Phi(p, \cdot): \bar{D} \rightarrow \bar{B}_{n}$ is one-one and onto.

It remains to prove the regularity of $\Phi$. Let $p \in \partial D, q \in \bar{D} \backslash\{p\}$ be fixed. Let $p(t)$ be a $C^{k}$ curve on $\partial D$ with $p(0)=p$, and $q(t)$ a $C^{k}$ curve in $\bar{D}$ disjoint from $p(t)$ with $q(0)=q$. Let $\varphi_{t}$ be a family of biholomorphic maps of $\mathbf{C}^{n}, C^{k-2}$ with respect to $t$, sending $p(t)$ to $(1,0, \ldots, 0), \nu(p(t))$ to $(1,0, \ldots, 0)$, and the complex Hessian of $D$ at $p(t)$ to $I_{n}$. Set $z_{t}=\varphi_{t}(q(t)), D_{t}=\varphi_{t}(D)$, and apply Proposition $5^{\prime}$ to $D_{t}$ and $z_{t}$; we obtain $E$-mappings $f_{t}$ of $D_{t}$ with $f_{t}(1)=(1,0, \ldots, 0), f_{t}\left(\xi_{t}\right)=z_{t}$ and $\left\langle f_{t}^{\prime}(1),(1,0, \ldots, 0)\right\rangle=\left\langle f_{0}^{\prime}(1),(1,0, \ldots, 0)\right\rangle$. Also we have $f\left(p(t), v_{p(t)}(q(t)), \cdot\right)=$ $\varphi_{t}^{-1} \circ f_{t} \circ M_{t}$ where $M_{t} \in \operatorname{Aut}(U)$ is to adjust the length of $\left(\varphi_{t}^{-1} \circ f_{t}\right)^{\prime}(1)$ making it an element of $L_{p(t)}$. Since $\xi_{t}, f_{t}$ are $C^{k-4}$ with respect to $t$ and $f_{t}^{\prime}(1)$ is $C^{k-5}$ with respect to $t$, we see that $M_{t}$ is $C^{k-5}$ with respect to $t$. Hence $v_{p(t)}(q(t))$, $\zeta_{p(t)}(q(t))$ and $f\left(p(t), v_{p(t)}(q(t)), \cdot\right)$ are all $C^{k-5}$ with respect to $t$. It follows that $v_{p}(q), \zeta_{p}(q), f\left(p, v_{p}(q), \cdot\right)$ are $C^{k-5}$ with respect to $p, q$, if $p \neq q$. Therefore, in view of $(17), \Phi(p, q) \in C^{k-5}(\partial D \times \bar{D} \backslash\{$ diagonal of $\partial D \times \partial D\})$. Moreover, for $p \in \partial D$ fixed, let $F_{p}$ be the inverse map of $\Phi(p, \cdot)$ and $z_{0}=\nu(p) /|\nu(p)|$. Then for $z \in \bar{B}_{n}$, $z \neq z_{0}$, it is easy to see that $F_{p}(z)=f(p, v, \zeta)$ where

$$
v=\left\langle\bar{z}_{0}-\bar{z}, z_{0}\right\rangle\left(z_{0}-z\right)\left|z-z_{0}\right|^{-2} \text { and } \varsigma=1-\left(\left|z_{0}-z\right|^{2} /\left\langle\bar{z}_{0}-\bar{z}, z_{0}\right\rangle\right) \text {. }
$$

It then follows that $\Phi(p, \cdot)$ is a $C^{k-5}$ diffeomorphism from $\bar{D} \backslash\{p\}$ to $\bar{B}_{n} \backslash\left\{z_{0}\right\}$. This completes the proof of Theorem 3.

\section{BIBLIOGRAPHY}

1. C. H. Chang, H. P. Lee and A. N. Wang, Extremal analytic discs and the chains, Chinese J. Math. 13 (1985), 239-255.

2. J. E. Fornaess, Embedding strictly pseudoconvex domains in convex domains, Amer. J. Math. 98 (1976), 529-569. 
3. J. Globevnik and E. L. Stout, The ends of discs, Bull. Soc. Math. France 114 (1986), 175-195.

4. G. M. Goluzin, Geometric theory of functions of a complex variable, Transl. Math. Monographs, vol. 26, Amer. Math. Soc., Providence, R.I. 1969; reprinted 1983.

5. H. Jacobowitz, Chains in CR geometry, J. Differential Geometry 21 (1985), 163-194.

6. L. Lempert, La métrique de Kobayashi et la représentation des domaines sur la boule, Bull. Soc. Math. France 109 (1981), 427-474.

7. _ Intrinsic distances and holomorphic retracts, Complex Analysis and Applications, 1981, Bulgar. Acad. Sci., Sofia, 1984, pp. 341-364.

8. E. A. Poletskii, The Euler-Lagrange equations for extremal holomorphic mappings of the unit disc, Michigan Math. J. 30 (1983), 317-333.

9. M. Suzuki, Complex geodesics on convex domains, Math. Rep. Toyama Univ. 9 (1986), 137-147.

10. A. G. Vitushkin, Global normalization of a real analytic surface along a chain, Soviet Math. Dokl. 27 (1983), 270-273.

Institute of Mathematics, ACademia Sinica, Taipei, Taiwan, R.O.C. (Current address of C.-H. Chang and H.-P. Lee)

Department of Mathematics, National Central University, Chung-Li, TaOYUAN, TAIWAN, R.O.C. (Current address of M. C. Hu) 